\title{
Modelling Two-Dimensional Flow Around a Circular Cylinder with Different Roughness Height
}

\author{
Jianbo Xie ${ }^{1, a}$, Xin Wang ${ }^{1, b}$ \\ ${ }^{1}$ Guangdong University of Technology, Guangzhou, China \\ axiejianbo1990@126.com, bxinwang@gdut.edu.cn
}

\begin{abstract}
Keywords: Roughness height, Vortex shedding, Drag coefficient, Lift coefficient, Pressure coefficient

Abstract. The present paper focuses on the analysis of the flow around a circular cylinder on the surface with different roughness height (Ks). In this simulation, an iced transmission line was simplified to a two-dimensional cylinder with roughness wall on the part of iced area and smooth wall on the remaining, the Reynolds number $(\mathrm{Re})$ is about 200, and the turbulence was modeled using the SST k- $\omega$ model. The variation tendency of lift coefficient $\left(\mathrm{C}_{1}\right)$ and drag coefficient $\left(\mathrm{C}_{\mathrm{d}}\right)$ were obtained as well as the mean pressure coefficient $\left(\mathrm{C}_{\mathrm{p}}\right)$ of the cylinder. The computational results indicated that pressure distribution on the cylindrical surface is asymmetry because of different roughness condition in iced and un-iced area and the separation point of vortex moves backward with the increase of the roughness height.
\end{abstract}

\section{Introduction}

The flow around a circular cylinder is a classic fluid mechanics problem. It is associated with a lot of practical applications in Civil Engineering field. For example, the action of water on bridges, the pillar of offshore drilling platform, the subsea transport pipeline, mooring line, etc, and the wind on tower building, chemical tower equipment, aerial cable, transmission lines, etc. Therefore, it has not only theoretical significance, but also obvious social and economic benefits to conduct further research to flow around a cylinder, understanding the flow mechanism.

It's easy for overhead conductor to produce ice covering its underlying surface in wet and cold weather conditions and difficult to determine the surface shape. The ice shapes of conductors are extremely diversity. Although many ice thickness estimation models were established based on the observation and mereorological data in different areas[1,2]. The current research on aerodynamic parameters of iced conductors can only choose the typical shapes, such as crescent shape[3, 4], fan shape[5] and D shape[6-8]. Wind tunnel test and numerical simulation are two common ways to mearsure the aerodynamic forces of the iced conductors. Chadha and Jaster[4] observed like the areodynamic coefficients varies with the angle of wind attack on conductors attached with crescent-shaped ice by wind tunnel test. In [6-8], aerodynamic characteristics of D-shaped conductors were investigated by means of wind tunnel test. Li et al.[5] researched the aerodynamic parameters of fan-shaped iced conductors with different thickness by numerical simulation. However, this simplication was idealized.

The present work is to study the flow around a circular cylinder for the roughness height. In this paper, four different types of roughness height were used to represent the surface roughness of iced conductor. The Reynolds number is about 200 when the cylindrical surface is assumed to be smooth. In our study, the roughness height is determined by the equivalent sand gravel roughness (Ks) measured by H.Schlichtin[9]. The value of Ks is shown as Table 1. The pressure distribution of the cylindrical surface and force coefficients are discussed in detail. 


\section{Simulation Computation}

Numerical Method. The governing equations for the unsteady flow of a two-dimensional incompressible viscous fluid around a circular cylinder are the classical continuity and Navier-Stokes equations. The turbulence was modeled using the SST k- $\omega$ model and PISO algorithm selected to solve the pressure-velocity coupling equations. Pressure differencing sheme is central differencing scheme. The second order upwind scheme was adopted for the spatial discretization of momentum, turbulent kinetic energy and turbulent dissipation rate. Second order implicit was employed for time-dependent solution formulations.

Computational Domain and Boundary Conditions. The analysis model in this study is shown in Figure 1. The size of computational domain is $37 \mathrm{D} \times 24 \mathrm{D}$, where $\mathrm{D}$ is the cylinder diameter which is seted to $0.0268 \mathrm{~m}$. The domain was divided into two parts, fluid_in and fluid_out. The show in Figure 1 does not represent the actual size. The fluid is air and its density is $1.225 \mathrm{~kg} / \mathrm{m}^{3}$, The inflow velocity is specified as $0.109 \mathrm{~m} / \mathrm{s}$, the turbulence intensity $\mathrm{I}$ is $5 \%$ at the inlet boundary. The right oundary is assigned an outflow boundary condition with normal gradients $\frac{\partial u}{\partial x}$ and $\frac{\partial v}{\partial y}$ set to zero, $u$ and $v$ are the velocity of the cylinder in the $\mathrm{x}$ and $\mathrm{y}$ direction. The upper and lower boundaries are assigned the symmetry boundary condition $\left(\frac{\partial u}{\partial y}=0, v=0\right)$. The upper part of the cylindrical surface is assumed to be smooth, while the lower part is set to be roughness wall shown in Figure 2.

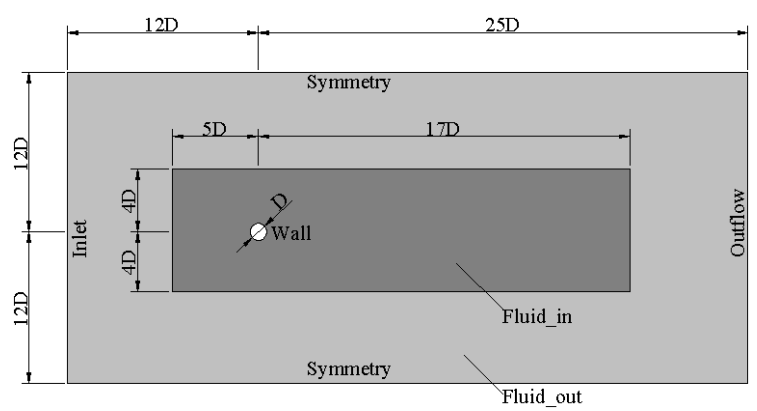

Figure 1 Analysis model and boundary conditions (cylinder is not drawn to scale)

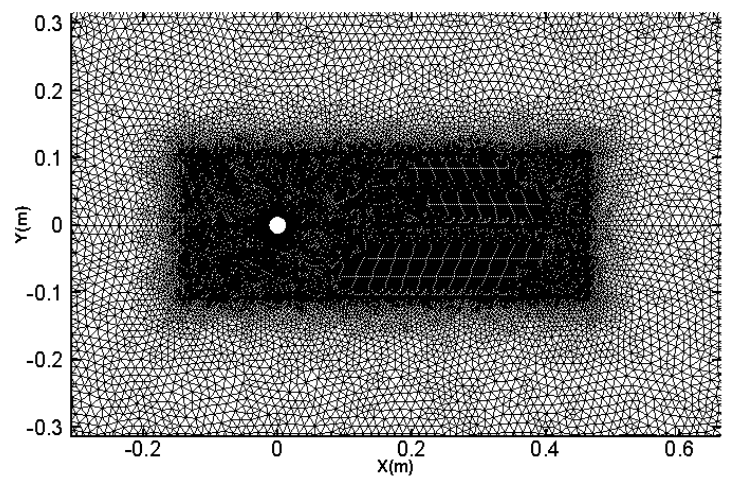

Figure 3 Mesh layout

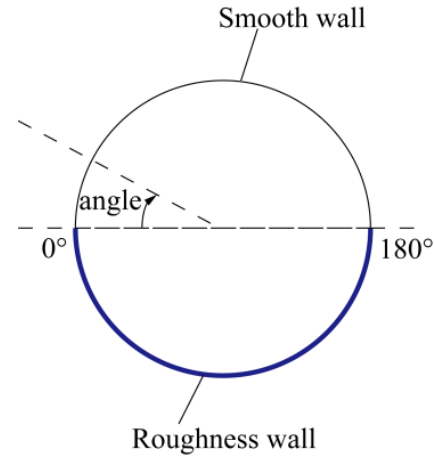

Figure 2 Cylindrical surface

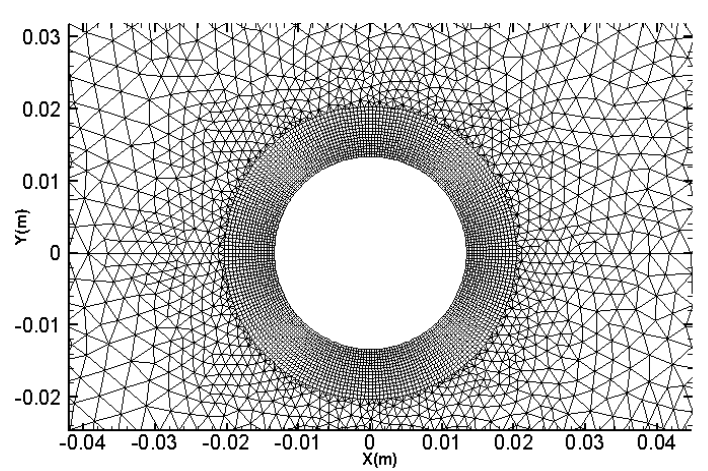

Figure 4 Mesh near the cylinder

Mesh Generation. The domain was divided into two parts, i.e. fluid_in and fluid_out, unstructured mesh was used. In fluid_in, the mesh was dense and the maximum mesh size was $0.003 \mathrm{~m}$. In fluid_out, the mesh gradually became sparser, the maximum mesh size was $0.012 \mathrm{~m}$, and the growth rate was 1.10 . The thickness of the first layer mesh met the condition that $\mathrm{y}^{+}=0.172 \frac{\Delta y}{\mathrm{~L}} \operatorname{Re}^{0.9}[10] \approx 1$, where $\Delta y$ is the distance from the wall to the cell center, $\mathrm{L}$ is the characteristic length of the cylinder 
and $\mathrm{L}$ is equal to $\mathrm{D}, \mathrm{Re}=\frac{\rho \mathrm{uL}}{\mu}$, where $\rho$ is the density, $\mathrm{u}$ characteristic velocity, $\mathrm{L}$ the characteristic length, $\mu$ dynamic viscosity. Here $\Delta y=0.0005 \mathrm{~m}$, the maximum number of the boundary layer was 16 , the growth rate was 1.05 , and there were 120 nodes on the surface of the cylinder. The total number of cells was 48194, the mesh layout is shown in Figure 3-4.

Aerodynamic parameters. The fluid past a circular cylinder will generate lift force in the $\mathrm{y}$-direction and drag force in the x-direction. Lift coefficient $\left(\mathrm{C}_{1}\right)$ and drag coefficient $\left(\mathrm{C}_{\mathrm{d}}\right)$ are normally used to reflect the lift and drag force, which are described as follows:

$$
\begin{aligned}
& C_{d}=\frac{F_{d}}{\frac{1}{2} \rho u^{2} \mathrm{~A}} . \\
& C_{1}=\frac{F_{1}}{\frac{1}{2} \rho u^{2} \mathrm{~A}} .
\end{aligned}
$$

Where $F_{d}$ and $F_{1}$ is the drag force and the lift force respectively, A the area of the upwind surface of the cylinder.

The Strouhal number (St) is used to represent the non-dimentional vortex shedding frequency, defined by

$$
\mathrm{St}=\frac{\mathrm{f}_{\mathrm{s}} \mathrm{L}}{\mathrm{u}} \text {. }
$$

Where $f_{s}$ is the vortex shedding frequency.

Mean pressure coefficient $\left(\mathrm{C}_{\mathrm{p}}\right)$, described as

$$
\mathrm{C}_{\mathrm{p}}=\frac{\overline{\mathrm{p}}-\mathrm{p}_{\infty}}{\frac{1}{2} \rho \mathrm{u}^{2}} \text {. }
$$

Where $\bar{p}$ is the time average pressure of some measuring point on the cylindrical surface, $p_{\infty}$ the inflow pressure.

\section{Results And Disscussion}

Pressure Coefficients $\left(\mathbf{C}_{\mathbf{p}}\right)$. The $\mathrm{C}_{\mathrm{p}}$ graph of simulations is presented in Figure 5. The variation tendency of $C_{p}$ is basically the same which may be noted for the four different roughness height Ks. At the point of $0^{\circ}$ which is called stagnatin point, the $C_{p}$ value is about 1 the maximum value of the $\mathrm{C}_{\mathrm{p}}$ graph, then the pressure coefficients gradually descrease along with the surface of the cylinder. The $C_{p}$ values remain the same when the angle is between $120^{\circ}$ and $240^{\circ}$. However, the maximum absolute values of the pressure coefficient in the lower part of the cylindrical surface increase with the increase of the roughness height Ks, and the maximum absolute values of $\mathrm{C}_{\mathrm{p}}$ reach 1.5 and 2.0 when $\mathrm{Ks}=1.49 \mathrm{~mm}$ and $3.65 \mathrm{~mm}$ respectively. The absolute values of $\mathrm{C}_{\mathrm{p}}$ in the upper part reduce slowly compared with $\mathrm{Ks}=0 \mathrm{~mm}$. Although the distribution of $\mathrm{C}_{\mathrm{p}}$ is different with the different $\mathrm{Ks}$ value, the separation point at about $110^{\circ}$ is almost unchanged in the upper part of the surface. In the lower part, the segaration point abviously moves backwards as the Ks increases, and when $\mathrm{Ks}=3.65 \mathrm{~mm}$, the segaration occurs at about $240^{\circ}$. Figure 6 presents vortex structure in one cycle of vortex shedding at different $\mathrm{Ks}, \mathrm{t} 0$ means a moment in the flow field, $\mathrm{T}$ is the vortex shedding cycle. When Ks is small, the separation point of vortex in the upper part is almost same with that in the lower part. As Ks increases to $3.65 \mathrm{~mm}$, a clear shift of separation point has occurred. The $\mathrm{C}_{\mathrm{p}}$ graph indicates that it will put off the vortex shedding in the lower part of the cylindrical surface when increase the roughness height, therefore the whole distribution of pressure will be changed compared with the smooth condition. 


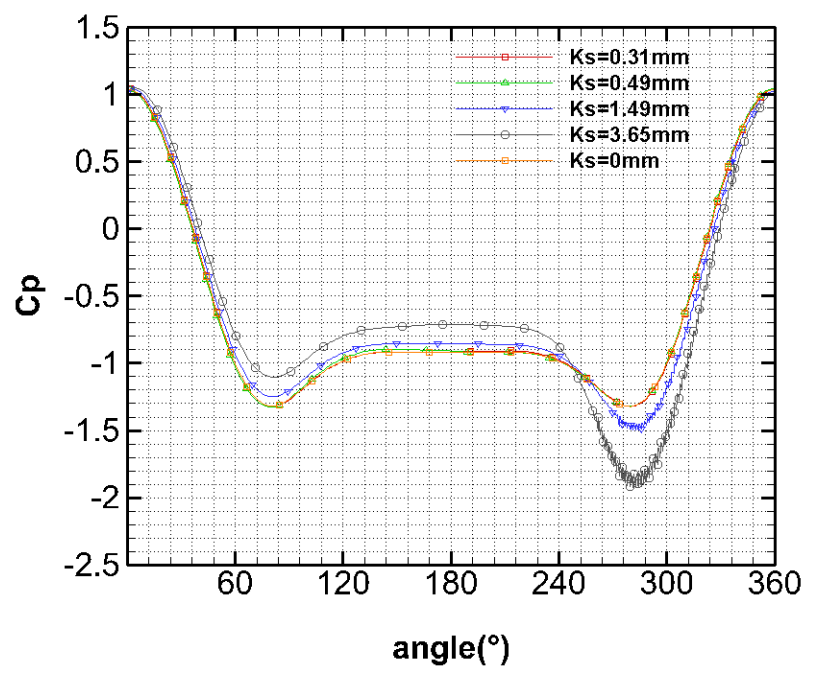

Figure 5 Distribution curve of $\mathrm{Cp}$

Drag and Lift Coefficient. As Figure 7 shows, the drag coefficient $\left(\mathrm{C}_{\mathrm{d}}\right)$ and lift coefficient $\left(\mathrm{C}_{\mathrm{l}}\right)$ oscillate around the center value after the

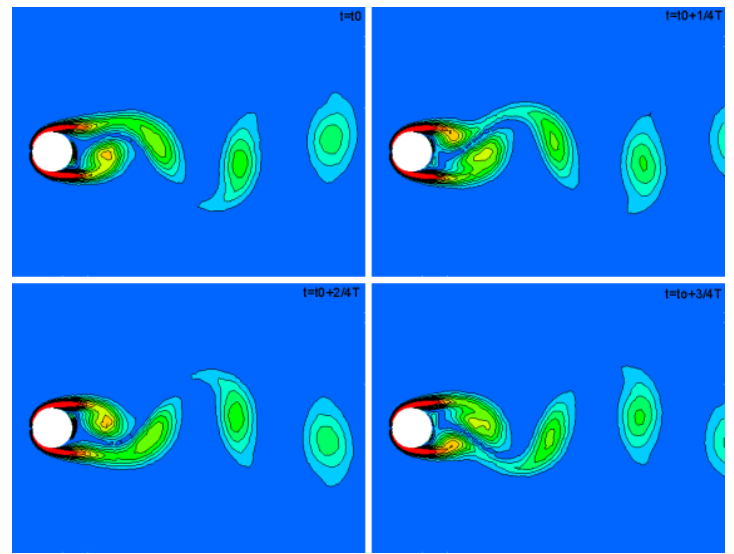

(a) $\mathrm{Ks}=.031 \mathrm{~mm}$

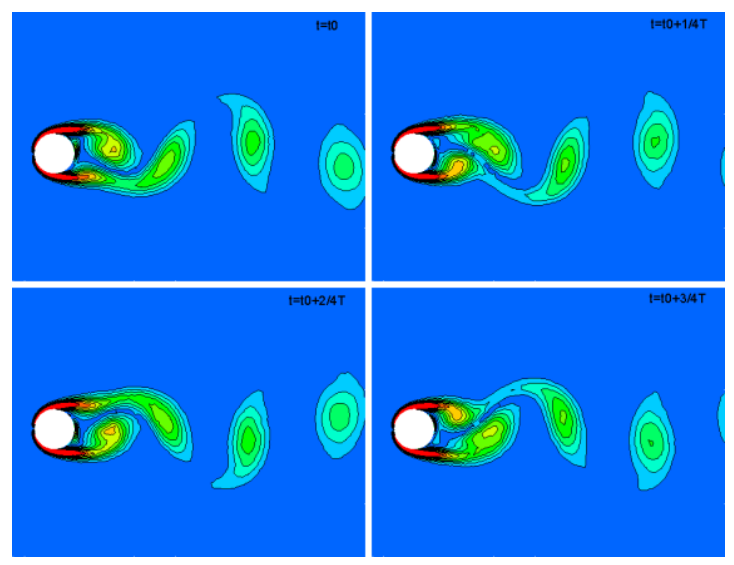

(c) $\mathrm{Ks}=1.49 \mathrm{~mm}$

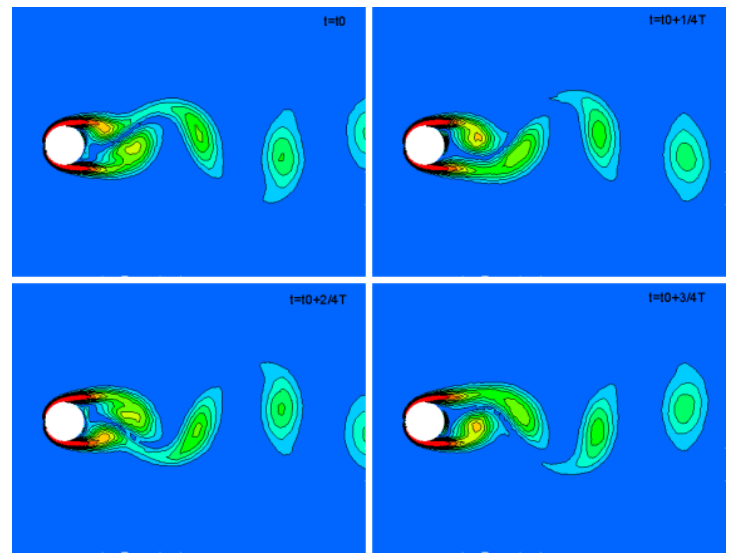

(b) $\mathrm{Ks}=0.49 \mathrm{~mm}$

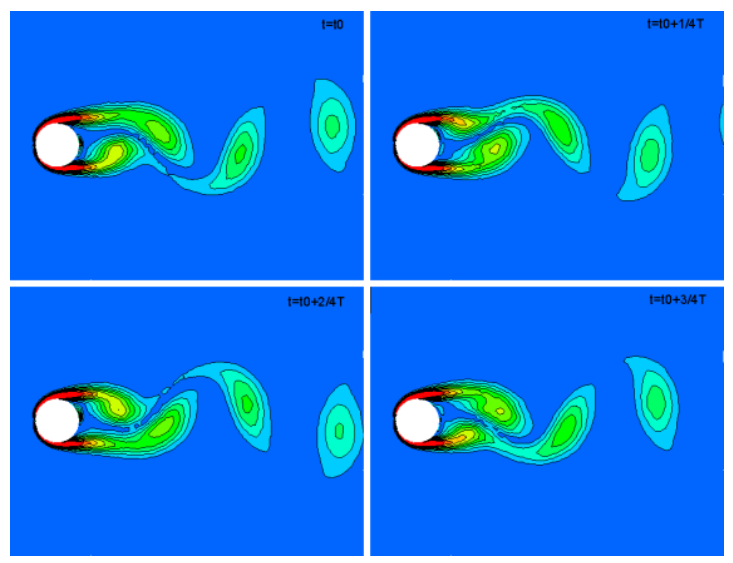

(d) $\mathrm{Ks}=3.65 \mathrm{~mm}$

Figure 6 Vortex structure in the wake

stability of the flow field. The mean values of $\mathrm{C}_{1}$ oscillate near $\mathrm{y}=0$ when $\mathrm{Ks}=0.31 \mathrm{~mm}$ and $0.49 \mathrm{~mm}$. Then the mean value of $\mathrm{C}_{1}$ starts to decrease and $\mathrm{C}_{1}$ has been negative when $\mathrm{Ks}$ increases to $3.65 \mathrm{~mm}$, that is to say that the aerodynamic lift force of the cylinder will no longer change direction and keep the negative direction unchanged. The vortex shedding frequency $\left(\mathrm{f}_{\mathrm{s}}\right)$ or the Strouhal number (St) gradually increases with the increase of Ks as Table 1 shows, but the average values of $\mathrm{C}_{\mathrm{d}}$ reduce little by little. This is because the drag force of the cylinder in the field is the result of de- 
viation of the pressure distribution, while the deviation is by the causes of boundary layer separation. The increase of roughness height is equivalent to increasing the turbulence of the boundary layer and put off the separation. As Figure 6 shows, the segaration point abviously moves backwards as the Ks increases in the lower part of the surface leads to the reduction of the adverse pressure gradient of wake, therefore, the pressure resistance diminishes at the same time. With regard to the bluff body such as cylinder, the pressure resistance is the main part of the drag force. Due to the difference between upper and lower surface roughness, the distribution of steady pressure presents the asymmetry.

Table 1 Force coefficient and vortex shedding frequency(RMS means root mean square)

\begin{tabular}{clccc}
\hline $\mathrm{Ks}$ & $\mathrm{RMS}\left(\mathrm{C}_{1}\right)$ & $\overline{\mathrm{C}_{\mathrm{d}}}$ & $\mathrm{f}_{\mathrm{s}}$ & $\mathrm{St}$ \\
\hline 0 & 0.383 & 1.302 & 0.649 & 0.160 \\
0.31 & 0.372 & 1.289 & 0.685 & 0.168 \\
0.49 & 0.368 & 1.279 & 0.695 & 0.171 \\
1.49 & 0.329 & 1.196 & 0.678 & 0.167 \\
3.65 & 0.406 & 1.010 & 0.700 & 0.172 \\
\hline
\end{tabular}

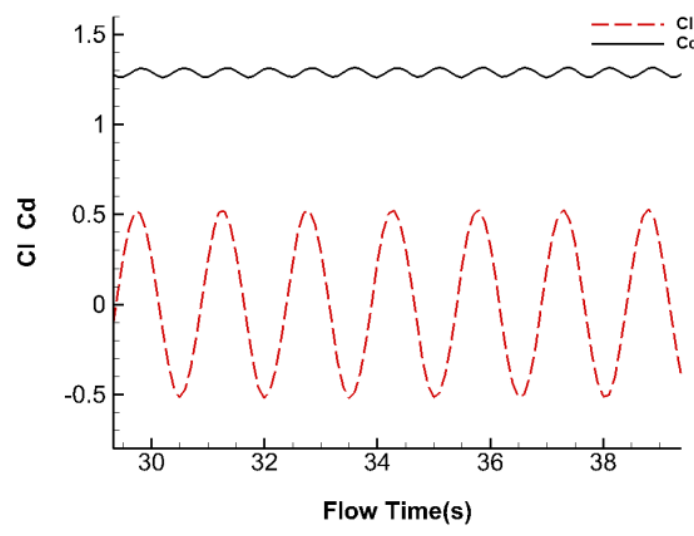

(a) $\mathrm{Ks}=0.31 \mathrm{~mm}$

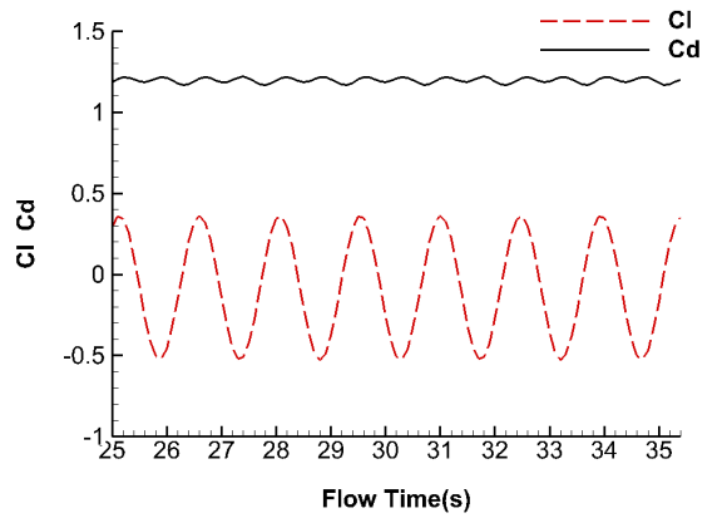

(c) $\mathrm{Ks}=1.49 \mathrm{~mm}$

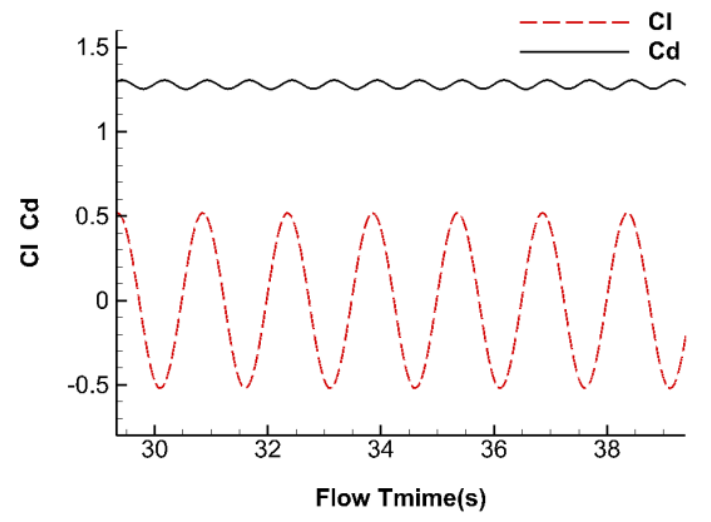

(b) $\mathrm{Ks}=0.49 \mathrm{~mm}$

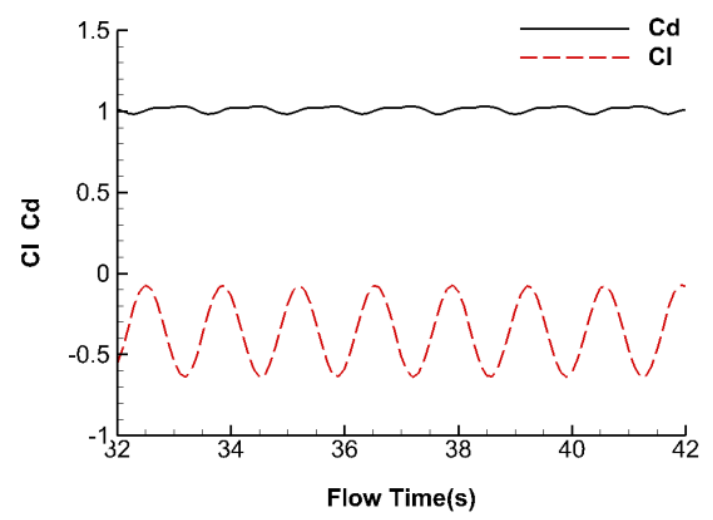

(d) $\mathrm{Ks}=3.65 \mathrm{~mm}$

Figure 7 Time history of drag and lift coefficient

\section{Conclusions}

This research presents a two-dimensional numerical study of a flow around circular cylinder Reynolds number $=200$ with different roughness height. The various trends of lift coefficient, drag coefficient and pressure distribution of the cylindrical surface are analyzed.

The present work indicates that modifications to the roughness height of the lower part of the cylindrical surface can produce significant changes to the pressure distribution of the surface. The $\mathrm{CP}_{\mathrm{P}}$ 
graph is no longer symmetry when the roughness of the lower part of the surface has been changed. It can be found that the maximum absolute values of $\mathrm{C}_{\mathrm{p}}$ in the upper part increase while that in the lower part decrease with the increase of Ks.

Drag coefficients significantly decrease for the cylinder with the increase of Ks because of the reduced of the adverse pressure gradient of wake. The mean values of lift coefficient no longer oscillate near $\mathrm{y}=0$ as the center line, and the lift coefficient will keep negative if the Ks is big enough.

\section{Acknowledgement}

This work was supported by National Natural Science Foundation of China (No. 51378128) and Natural Science Foundation of Guangdong Province, China (No. 2015A030313498).

\section{References}

[1] F. Porcú, E. Smargiassi and F. Prodi, "2-D and 3-D modelling of low density ice accretion on rotating wires with variable surface irregularities," Atmospheric Research, vol. 36, pp. 233-242, 1995.

[2] L. Makkonen, "Modeling power line icing in freezing precipitation," Atmospheric Research, vol. 46, pp. 131-142, 1998.

[3] R. Keutgen and J. L. Lilien, "Benchmark Cases for Galloping with Results Obtained from Wind Tunnel Facilities - Validation of a Finite Element Model," IEEE Transactions On Power Delivery, vol. 15, no. 1, pp. 367-374, 2000.

[4] J. Chadha and W. Jaster, "Influence of turbulence on the galloping instability of iced conductors," IEEE Transactions on Power Apparatus and Systems, vol. PAS-94, no. 5, pp. 1489-1499, 1975.

[5] X. M. Li, K. J. Zhu and B. Liu, "numerical and Experimental Simulation of Aerodynamic Characteristics of Typical Iced Conductor," High Voltage Engineering, vol. 40, no. 2, pp. 427-433,2014.

[6] P. Van Dyke and A. Laneville, "Galloping of a single conductor covered with a D-section on a high-voltage overhead test line," Journal of Wind Engineering and Industrial Aerodynamics, vol. 96, no. 6-7, pp. 1141-1151, 2008.

[7] J. Chadha and W. Jaster, "Influence of turbulence on the galloping instability of iced conductors," IEEE Transactions on Power Apparatus and Systems, vol. PAS-94, no. 5, pp. 1489-1499, 1975.

[8] Y. M. DESAI, P. YU, A. H. SHAH and N. POPPLEWELL, "Pertubation-based Finite Element Analyses Of Transmission Line Galloping," Journal of Sound and Vibration, vol. 191, no. 4, pp. 469-489, 1996.

[9] H. Schlichting, Boundary Layer Theory. 7st ed, McGraw-Hill Book Company, 1979, ch. 21.

[10] S. P. Chang, Y. S. Wang and Z. Y. Pang, "Numerical simulation of flow around circular cylinder using SST DES model," Ship Sci \& Technol, vol. 31, no. 2, pp. 30-33, 2009. 